\title{
Neuer Masterstudiengang: Multiple Sklerose Management
}

\author{
Isabel Voigt · Christine Stadelmann · Sven G. Meuth · Bernhard Schipp · Peter Flachenecker · Mathias Mäurer · \\ Richard H. W. Funk · Franziska Ramisch · Joachim Niemeier · Tjalf Ziemssen (D)
}

Eingegangen: 5. Oktober 2021 / Angenommen: 25. November 2021 / Online publiziert: 2. Februar 2022

(C) The Author(s), under exclusive licence to Springer-Verlag GmbH Austria, ein Teil von Springer Nature 2022

Zusammenfassung Der neue Studiengang „Multiple Sklerose Management" richtet sich an Ärzt*innen, Therapeut*innen, Pflegepersonal, Wissenschaftler*innen, Apotheker*innen, Psycholog*innen und Biolog*innen, die sich im Bereich der Multiplen Sklerose (MS) spezialisieren wollen. Nach erfolgreicher Akkreditierung im Jahr 2019 befindet sich die erste Matrikel seit 2020 in dem von der Dresden International University (DIU) angebotenen Masterstudiengang. Über eine Dauer von 4 Semestern kann er berufsbegleitend und größtenteils digital absolviert werden. Der Masterstudiengang gliedert sich in 6 Module mit den

\section{Voigt · T. Ziemssen $(\bowtie)$}

Zentrum für klinische Neurowissenschaften, Neurologische Klinik, Universitätsklinikum Carl Gustav Carus Dresden, Technische Universität Dresden, Dresden, Deutschland Tjalf.Ziemssen@ukdd.de

\section{Stadelmann}

Institut für Neuropathologie, Universitätsklinikum Göttingen, Göttingen, Deutschland

\section{S. G. Meuth}

Neurologische Klinik, Medizinische Fakultät,

Universitätsklinikum Düsseldorf, Düsseldorf, Deutschland

\section{B. Schipp}

Professur für quantitative Verfahren, insb. Ökonometrie, Fakultät Wirtschaftswissenschaften, Technische Universität Dresden, Dresden, Deutschland

\section{P. Flachenecker}

Neurologisches Rehabilitationszentrum Quellenhof, Bad Wildbad, Deutschland

\section{Mäurer}

Neurologie und Neurologische Frührehabilitation, Klinikum Würzburg Mitte, Juliusspital, Würzburg, Deutschland

R. H. W. Funk · F. Ramisch $\cdot$ J. Niemeier Dresden International University (DIU), Dresden, Deutschland
Schwerpunkten Grundlagen, Klinik und Diagnostik, MS-Studien und Statistik, krankheitsmodifizierende und symptomatische Therapie, Krankheitsmonitoring und Dokumentation. Der Unterricht beinhaltet theoretische Anteile und zahlreiche Praxiseinheiten. Ein weiteres Ziel ist es, aus wissenschaftlichen Publikationen und klinischen Studien therapeutische Interventionspläne und Problemlösungsstrategien abzuleiten, sie weiterzuentwickeln und in der Patientenversorgung anzuwenden. Die Dozent*innen stammen aus Deutschland, Österreich und der Schweiz und sind überwiegend Professor*innen. Die Schirmherrschaft für den Studiengang übernimmt die Deutsche Multiple Sklerose Gesellschaft. Dieser Beitrag stellt den Studiengang im Detail vor.

Schlüsselwörter Neurologie · Studium · Ausbildung • Spezialisierung · Dresden International University

\section{New master's program: multiple sclerosis management}

Summary The new study program "Multiple Sclerosis Management" is aimed at physicians, therapists, nurses, scientists, pharmacists, psychologists and biologists who want to specialize in the field of multiple sclerosis (MS). After successful accreditation in 2019, the first students have been in the master's program offered by Dresden International University (DIU) since 2020. Over a period of four semesters, it can be completed part-time and largely digitally. The master's program is divided into six modules focusing on basics, clinical and diagnostic aspects, MS studies and statistics, disease-modifying and symptomatic therapy, disease monitoring and documentation. The teaching includes theoretical parts and numerous practical units. A further goal is to derive therapeutic intervention plans and problem-solving strategies 
from scientific publications and clinical studies, to develop them further and to apply them in patient care. The lecturers come from Germany, Austria and Switzerland and are predominantly professors. The German Multiple Sclerosis Society is the patron of the course. This article presents the study program in detail.

Keywords Neurology · Education - Qualification · Specialization · Dresden International University

\section{Warum Multiple Sklerose Management?}

Multiple Sklerose (MS) ist eine chronische neurologische Erkrankung, die im jungen Erwachsenenalter beginnt und ein Leben lang andauert, ohne dass sich die Lebenserwartung der Patient*innen verringert [1]. Neben ihrem chronischen und/oder episodischen Verlauf hat die MS einen heterogenen Charakter und verschiedene Dimensionen, sodass das klinische Bild sehr vielfältig ist. $\mathrm{Zu}$ den neurologischen Symptomen zählen u.a. Muskelschwäche, Sensibilitätsstörungen, Fatigue, Gleichgewichtsstörungen, Spastik und Sehstörungen [2]. Infolge der Heterogenität der Symptome und der Verlaufsform ist eine Prognose zu Beginn der Erkrankung nur schwer möglich. Jedoch können heutzutage immer mehr Betroffene ein weitgehend normales Leben führen, da es in den letzten Jahren bedeutende Innovationen bei der Diagnose und Behandlung der MS gegeben hat [3, 4]. Folglich sollten MS-Spezialist*innen mit dem „State of the Art Management" sowohl der MS im Speziellen als auch der chronisch entzündlichen Erkrankungen des zentralen Nervensystems (ZNS) im Allgemeinen vertraut sein. Die Ausbildung von MS-Spezialist*innen stellt einen wichtigen Baustein im Kampf gegen die MS dar.

Bislang existierte jedoch kein strukturiertes und industrieunabhängiges Fortbildungsprogramm rund um die MS. Bestehende Kurse konzentrierten sich entweder auf breitere Spektren wie Neurowissenschaften und Neurodegeneration [5], Immunologie und entzündliche Erkrankungen sowie Neuroimmunologie [6], sie richteten sich an eine bestimmte Zielgruppe, wie z.B. Physiotherapeut*innen [7] und MS-Betreuende [8], oder es wurden nur Teilaspekte der MS in Einzelkursen behandelt [9]. Von Pharmaunternehmen gesponserte spezifische Weiterbildungsprogramme gelten als nicht unabhängig.

So entstand die Idee, einen eigenen Masterstudiengang für die Ausbildung zu MS-Spezialist*innen zu konzipieren. Ein solcher Studiengang, der sich auf eine einzige Krankheitsentität konzentriert, stellt ein Novum innerhalb der Landschaft medizinischer (Weiterbildungs-)Studiengänge dar. Unter der Federführung eines Gremiums von MS-Expert*innen und dem erfahrenen Team der Dresden International University (DIU) entstand der bisher in dieser Form einzigartige Masterstudiengang „Multiple Sklerose Management“ (MSM) [10]. Er bietet ein vollständiges und industrieunabhängiges Gesamtpaket rund um die MS und lässt sich somit von anderen wissenschaftlichen Zeitschriften oder Weiterbildungsangeboten abgrenzen, die meist nur einen sehr kleinen Aspekt der Pathologie, der Symptome oder der Behandlung und Pflege abdecken.

\section{Zielgruppe und Qualifikationsziele}

Der berufsbegleitende deutschsprachige Masterstudiengang „Multiple Sklerose Management" richtet sich an Ärzt*innen, Therapeut*innen, Pflegepersonal und Wissenschaftler*innen in Klinik, Wissenschaft und Praxis, in der Pharmaindustrie und öffentlicher Anstellung sowie Apotheker*innen, Psycholog*innen und Biolog*innen, die sich auf MS spezialisieren wollen. Sie sollen die Fähigkeit zu einer ausführlichen wissenschaftlich fundierten Diagnostik bzw. Differenzialdiagnostik bei Verdacht auf chronisch entzündliche Erkrankungen des ZNS, insbesondere der MS, erhalten. Im Weiteren sollen sie mithilfe umfangreicher Kenntnisse verschiedener Medikamente und Therapiestrategien die aktuellsten und am besten geeigneten Therapiemaßnahmen und -strategien für die individuellen Patient*innen ergreifen und dabei stets ein detailliertes und engmaschiges Monitoring der Krankheitsaktivität verfolgen können. Neben der praxisorientierten Anwendung von detailliertem und aktuellem Fachwissen zu Grundlagen, Klinik und Diagnostik, Therapie, Monitoring und Dokumentation sowie Studien und Statistik im Bereich MS erlernen die Absolvierenden das bedarfsgerechte Gestalten und Modifizieren von therapeutischen Interventionen im stationären sowie ambulanten Bereich, ausgerichtet an der Komplexität des Versorgungsauftrags. Sie erlangen ausgeprägte Kompetenzen zum Transfer von theoretischem Wissen in die Praxis, zur interdisziplinären, vernetzten Zusammenarbeit mit anderen relevanten Berufsgruppen des Gesundheits- und Sozialsystems, zur intra- und interdisziplinären und sektorenübergreifenden Versorgungssteuerung und zum Schnittstellenmanagement sowie Kompetenzen im Bereich der Kommunikation zwischen Ärzt*innen und Patient*innen und beim Coaching und der Rehabilitation von Patient*innen. Ein übergeordnetes Ziel des Studiengangs besteht zudem darin, wissenschaftlich fundierte Urteile von wissenschaftlichem Regelwissen und Daten aus wissenschaftlichen Studien abzuleiten, sie im Kontext der individuellen Situation der Patient*innen $\mathrm{zu}$ interpretieren und darauf aufbauend therapeutische Interventionspläne und Problemlösungsstrategien $\mathrm{zu}$ erarbeiten und weiterzuentwickeln sowie in der direkten Patient*innenversorgung anzuwenden.

\section{Entstehungsgeschichte}

Nach ersten Gesprächen zur Kooperation des Zentrums für klinische Neurowissenschaften am Univer- 
Abb. 1 Schirmherrschaft der DMSG, v.l.: Prof. Richard H.W. Funk (DIU), Prof. Judith Haas (DMSG), Prof. Tjalf Ziemssen (UKD). (Abdruck mit freundlicher Genehmigung des Universitätsklinikums Dresden)

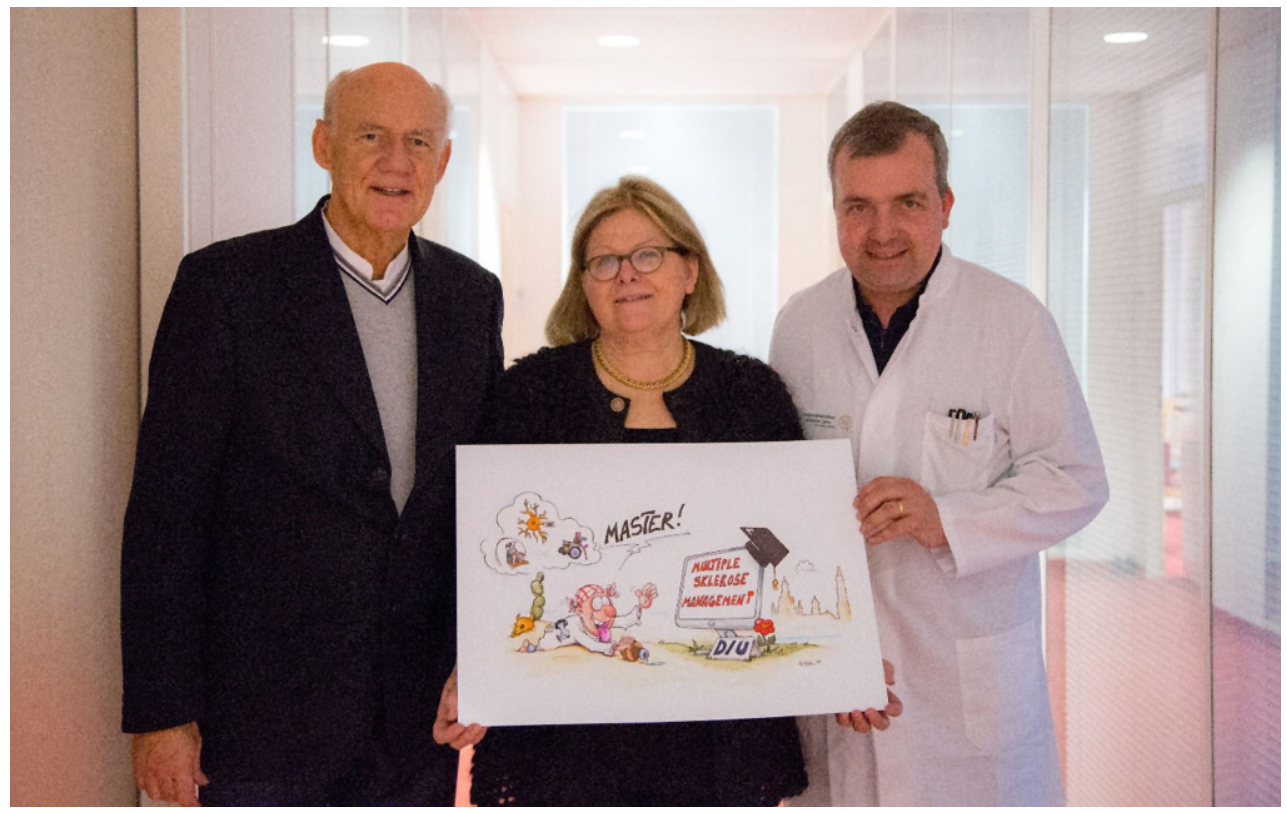

sitätsklinikum Dresden (UKD) mit der DIU starteten 2018 die Planungen für den Studiengang. Innerhalb der wissenschaftlich-fachlichen Leitung fanden Gespräche zur inhaltlichen und formellen Konzeption statt, und die für die Akkreditierung notwendigen Unterlagen wurden vorbereitet. Anfang 2019 konnte die Deutsche Multiple Sklerose Gesellschaft (DMSG) als Schirmherrin für den Studiengang gewonnen werden (Abb. 1). Als Kooperationspartner folgten die Deutsche Gesellschaft für Neurologie (DGN), der Berufsverband Deutscher Nervenärzte (BVDN), der Berufsverband Deutscher Neurologen (BDN), die ParadigMS Foundation und die European Multiple Sclerosis Platform (EMSP).

Die Auswahl der Dozent*innen folgte den Kriterien der Expertise auf dem Gebiet der MS, der Reputation im wissenschaftlichen medizinisch-fachlichen Rahmen und bisherigen Publikationen. Knapp 90\% der Dozent*innen sind Professor*innen oder professorale Dozent*innen, ca. $10 \%$ sind Praxisdozent*innen oder wissenschaftliche Mitarbeiter*innen. Die Expert*innen stammen aus Deutschland, Österreich und der Schweiz und verfügen über praktische Erfahrungen der Anwendbarkeit von Wissen sowie der situationsgerechten und effizienten Vermittlung von Wissen.

Im ersten Quartal 2019 wurden die Dokumente für die Akkreditierung bei der Akkreditierungsagentur ACQUIN eingereicht. Im dritten Quartal 2019 fand ein erstes Treffen der Dozent*innen beim Kongress der Deutschen Gesellschaft für Neurologie in Stuttgart statt. Für die inhaltliche Entwicklung der einzelnen Module wurden Modulkoordinator*innen eingesetzt, die sich mit allen Dozent*innen mehrmals persönlich und online über die inhaltliche und konzeptionelle Gestaltung des Studiengangs austauschten. Sie wähl- ten im Team die Dozent*innen aus, spezifizierten die Lehrinhalte und ordneten sie den Lehrformaten zu. Gemeinsam mit der Programmleitung des Masterstudiengangs erarbeiteten sie auch die konkrete Zeitund Unterrichtsplanung. Im Oktober 2019 fand eine Schnuppervorlesung für Interessierte statt, und Ende des Jahres 2019 wurde der Studiengang akkreditiert.

Bereits seit Beginn der Akkreditierungsphase wurde der Studiengang auf den Internetseiten der DIU, mit einer Broschüre der DIU sowie mit mehreren Pressemitteilungen, kurzen Artikeln in Fachzeitschriften sowie einem Kongressposter beworben [11-15], womit auch die Rekrutierung der Bewerber*innen startete. Nach erfolgter Akkreditierung konnten sich die Interessierten dann für den Studiengang bewerben. Aufgrund der COVID-19-Pandemie musste der Start des Studiengangs von April 2020 auf Oktober 2020 verschoben werden (Abb. 2).

\section{Zulassungsvoraussetzungen}

Der Studiengang richtet sich mit seinem Abschluss „Master of Science“ (M.Sc.) nach den Anforderungen des Qualifikationsrahmens für Deutsche Hochschulabschlüsse vom 21.04.2005. Dies geht aus der Modularisierung und aus seiner Bewertung mit 60 Leistungspunkten auf der Stufe der ländergemeinsamen Strukturvorgaben hervor. Zum Studium zugelassen werden Bewerber*innen, die die entsprechenden Zulassungsvoraussetzungen erfüllen (Hochschulabschluss oder 240 ECTS). Diese sind in der Prüfungsordnung des Studiengangs beschrieben und richten sich nach Stufe 1 des Qualifikationsrahmens für Deutsche Hochschulabschlüsse vom 21.04.2005. Die Auswahl der Bewerber*innen erfolgt gemäß den Bestimmungen der Prüfungsordnung als Einzelprüfung in Form eines 


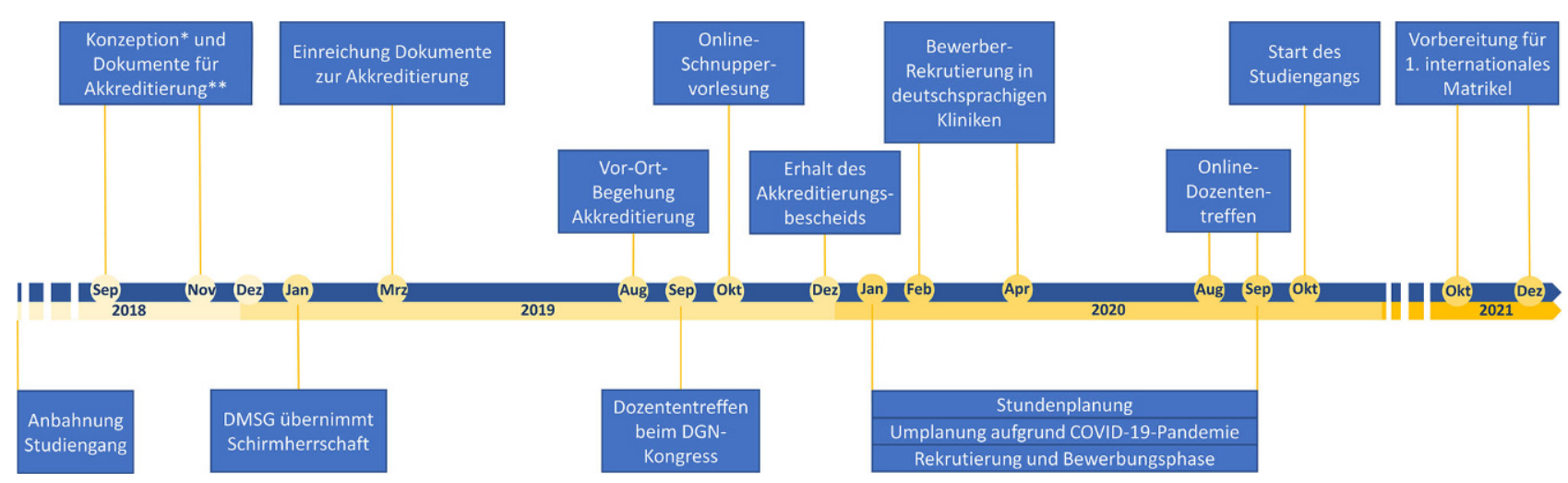

Abb. 2 Etablierung des Studiengangs im Zeitverlauf. *Festlegung der Module, Inhalte, Praxiseinheiten, Lehrformen, Modulverantwortlichen, Kooperationspartner und weiteres Ausarbeiten der Dokumente für die Akkreditierung sowie Beratung und Festlegung von eLearning-Elementen. ${ }^{*}$ Beschrei-

bung des Studiengangs (Qualifikationsziele, Studiengangsaufbau, -inhalte, Studierbarkeit, Implementierung, Kooperationspartner, Qualitätssicherung), Modulbeschreibungen, Studienverlaufsplan, Modulübersichtstabelle, Ablaufplan pro Semester, Liste der Lehrenden

\section{Masterstudiengang Multiple Sklerose Management}

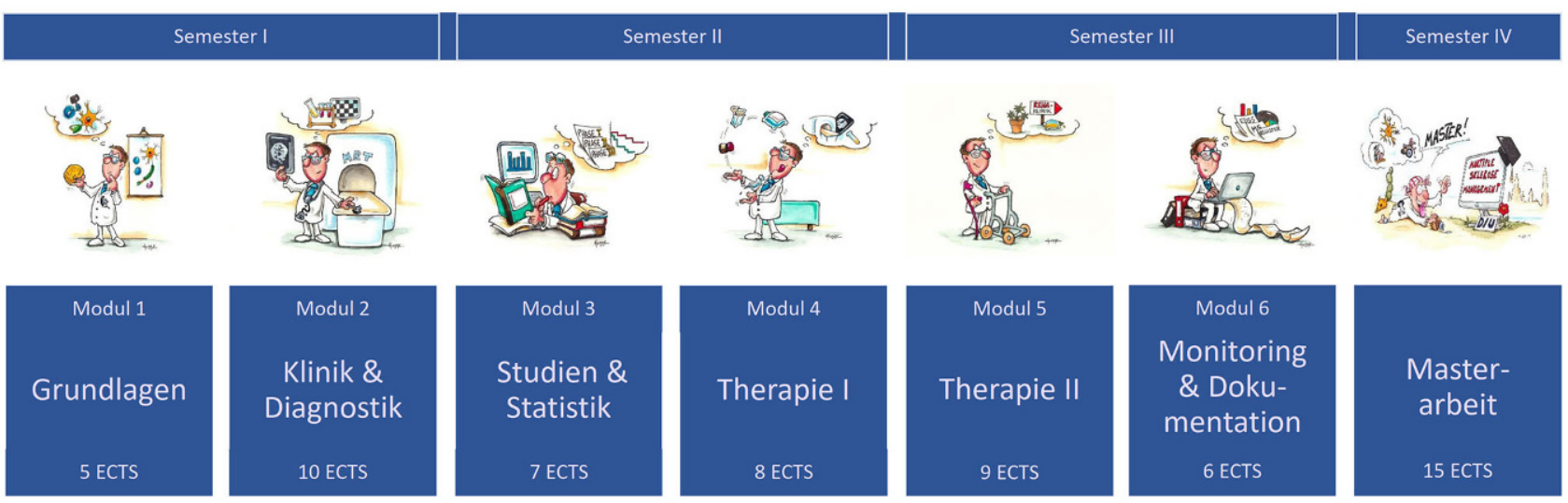

Abb. 3 Zeitplan für Module im MSM-Masterstudiengang mit Angabe der ECTS-Punkte; Cartoons: Phil Hubbe

Zulassungsgespräches durch eine vom Prüfungsausschuss für den jeweiligen Bewerbungszeitraum eingesetzte Zulassungskommission. Von dem Erfordernis des Zulassungsgespräches kann in definierten Einzelfällen abgesehen werden. Grundsätzlich kann aber nur zugelassen werden, wer einen Abschluss in einem Gesundheitsfachberuf vorweisen und einen Hochschulabschluss nachweisen kann. Die Bewerbungsunterlagen müssen vollständig schriftlich eingereicht werden. Die Zulassungskommission prüft die Unterlagen auf Vorliegen der Zulassungsvoraussetzungen und kontaktiert die Bewerber*innen. Zusätzlich besteht die Möglichkeit, bei der DIU ein Teilstipendium zu beantragen.

\section{Studiengangsaufbau}

Der berufsbegleitende Masterstudiengang „Multiple Sklerose Management" dauert 4 Semester und unterteilt sich in 6 Module (Abb. 3). Dazu gehören die 3 allgemeinen Grundlagenmodule MS Grundlagen, MS Klinik, Diagnostik und Praxis und MS Studien und Statistik sowie die 3 vertiefenden therapeutischen Module MS Therapie I und Praxis, MS Therapie II und Praxis sowie MS Monitoring, Dokumentation und Praxis inklusive Hospitationen in ausgewählten Zentren, Fallkonferenzen, Kongressteilnahmen und die Masterarbeit.

Der erste Teil des Masterstudiums (Semester 1 bis 3) umfasst 450 Präsenzstunden und besteht aus Präsenz- und Onlineveranstaltungen sowie Selbststudienphasen in 6 Modulen. Die Veranstaltungen finden in der Regel wöchentlich am Dienstagabend sowie im Blockunterricht am Wochenende teils online und teils in Präsenz statt. Der zweite Teil des Masterstudiums (Semester 4) dient der Anfertigung und Verteidigung (Kolloquium) der Masterarbeit.

Die Lehrveranstaltungen im Umfang von 45 Leistungspunkten und die Erstellung der Masterarbeit mit Disputation von 15 Leistungspunkten erstrecken 
sich über 4 Semester. Die Masterprüfung umfasst 2 Teile. Der erste Teil der Masterprüfung besteht aus Prüfungsleistungen zu den Modulen 1 bis 6 des Studiengangs (Modulprüfungen in Form von Klausuren, mündlichen Prüfungen und Fallberichten) und umfasst insgesamt 45 Leistungspunkte. Der zweite Teil der Masterprüfung beinhaltet die Anfertigung der Masterarbeit einschließlich der Verteidigung (Kolloquium). Für diesen zweiten Teil werden 15 Leistungspunkte erworben. Nach erfolgreicher Teilnahme wird der Titel „Master of Science“ (M.Sc.) verliehen.

\section{Studiengangsinhalte}

\section{Modul 1: MS Grundlagen}

In Modul 1 werden Grundlagen zum Krankheitsbild, zur Epidemiologie sowie zu unterschiedlichen Verläufen der MS mit ihren prognostischen Implikationen gelehrt. Es wird zudem umfangreiches Wissen zu formal-, molekular- und pharmakogenetischen Aspekten sowie zum Einfluss von Umweltaspekten vermittelt, sodass wichtige Einflussfaktoren für die Diagnosestellung und Therapie der individuellen Patient*innen abgeschätzt werden können. Die Kenntnis der immunologischen Grundlagen ist eine entscheidende Basis für das Verständnis der therapeutischen Interventionen, die im Rahmen folgender Module besprochen werden. Eine weitere Basis liefern die Kenntnisse zur Pathologie und Pathophysiologie, die zum einen einen wichtigen Stellenwert insbesondere für die symptomatische Therapie haben, zum anderen für das Verständnis von Bildgebung der MS eine wichtige Rolle spielen. Die Kenntnis dieser Grundlagen ist entscheidend für den klinischen Transfer, der erst nach dem Verständnis dieser Inhalte möglich wird.

\section{Modul 2: MS Klinik, Diagnostik und Praxis}

Zentrales Lernziel in diesem Modul ist es, eine weiterführende Diagnostik und Differenzialdiagnostik bei Verdacht auf chronisch entzündliche ZNS-Erkrankungen erstellen zu können, d.h. die Symptome der MS zu erkennen und diese nach ausführlicher Anamnese zu beurteilen. Dabei wird auch die Rolle von Liquor- und Blutuntersuchungen intensiv beleuchtet. Der Stellenwert bilddiagnostischer Verfahren wird anhand der Vorlesungen und Workshops zur Magnetresonanztomographie (MRT) und zur optischen Kohärenztomographie (OCT) im Detail deutlich. Es werden zudem Kenntnisse neurophysiologischer, neuropsychologischer und neurourologischer Untersuchungsverfahren vermittelt, um die Auswirkungen von Demyelinisierungen funktionell beurteilen zu können. Die erlernten diagnostischen und differenzialdiagnostischen Fertigkeiten werden durch die praktische Anwendung in Fallkonferenzen gefestigt.

\section{Modul 3: MS Studien und Statistik}

In Modul 3 werden die Grundlagen der klinischen Forschung und des Prozesses der Formulierung einer wissenschaftlichen Fragestellung vermittelt. Hierbei spielen besonders die bei der MS verwendeten Studiendesigns eine wichtige Rolle, deren unterschiedliche Endpunkte nach Durchlaufen dieses Moduls bekannt sein werden und angewendet werden können, d. h. es kann die richtige Methode passend $\mathrm{zu}$ einer bestimmten klinischen Forschungsfrage ausgewählt und auf Beispiele aus der MS Praxis angewendet werden. Neben den Grundlagen der Statistik stehen das Kennenlernen sowie die Auswahl und die Ergebnisinterpretation verschiedenster statistischer Tests im Fokus, um diese entsprechend dem Forschungsdesign und der Forschungshintergründe anwenden und diskutieren $\mathrm{zu}$ können. Besonderer Wert wird auch auf die Fallzahlplanung und Festlegung der Studienendpunkte gelegt. Eine weitere Säule des Moduls ist die Analyse und Interpretation von sog. Real-World-Daten, die im Laufe von Phase-IV-Registerstudien aggregiert werden. Da Modul 3 einen Schwerpunkt auf die Planung, Durchführung und Auswertung solcher Studien setzt, werden Fähigkeiten zu Biasreduktion und Matching insbesondere bei Real-World-Daten, wie z. B. das Propensity Matching, vermittelt.

\section{Modul 4: MS Therapie I und Praxis}

In diesem Modul werden die grundsätzlichen Unterschiede zwischen der Therapie akuter Schübe und einer krankheits- bzw. verlaufsmodifizierenden Therapie („disease modifying therapy“ [DMT]) der MS im Detail behandelt, um diese in der Praxis anwenden und Kolleg*innen als auch Fachfremden einschließlich Patient*innen erklären zu können. Es wird fundiertes und aktuelles Wissen zu Wirksamkeits- und Nebenwirkungsprofil der unterschiedlichen Medikamente sowie zu unterschiedlichen Therapiestrategien und -sequenzen vermittelt, sodass der Wirkmechanismus und die Sicherheit verschiedener Medikamente eingeschätzt und deren Einsatz im Einzelfall je nach Indikation und Patient*innenprofil abgewogen werden kann. Dies ist für eine erfolgreiche auf die Patient*innen zugeschnittene Therapiestrategie, die die Krankheitsaktivität reduziert und den Krankheitsverlauf günstig beeinflusst, von großer Bedeutung. So können auch zukünftige Therapiestrategien erarbeitet und wichtige Kenntnisse zu zukünftigen Strategien und aktuellen Studienansätzen angewendet werden. Gefestigt werden die Fertigkeiten durch die praktische Anwendung im Rahmen von Fallkonferenzen und einer Hospitation, wobei praktische Therapieentscheidungen vermittelt werden, die individualisiert für die Patient*innen hergeleitet werden sollen. 


\section{Modul 5: MS Therapie II und Praxis}

Modul 5 beschäftigt sich mit den verfügbaren nichtmedikamentösen und medikamentösen Verfahren zur Behandlung krankheitsassoziierter Symptome und deren bedarfsgerechtem Einsatz in der Praxis. Dies ist von hoher Relevanz, da eine Vielzahl von MSassoziierten Symptomen auftreten kann. Grundlage dafür bildet die Erlangung umfangreicher Kenntnisse $\mathrm{zu}$ Zielen und Durchführung symptomatischer und komplementärer Therapien mit ihren Indikationen und ihrem Risk-Benefit-Profil. Zudem wird in diesem Modul auch umfangreiches Wissen zu rehabilitativen Maßnahmen und palliativmedizinischen Aspekten vermittelt, um dazu beizutragen, durch den Einsatz neurokognitiver und psychologischer Interventionen die soziale Teilhabe und Selbstbestimmung der Patient*innen $\mathrm{zu}$ fördern und damit deren Lebensqualität zu verbessern. Durch einen umfangreichen Praxisanteil (Hospitation, Kongressteilnahme, Fallkonferenzen) werden die gelernten Inhalte vertieft und gefestigt, sodass MS Patient*innen in unterschiedlichen Krankheitsstadien optimal symptomatisch therapiert werden können.

\section{Modul 6: MS Monitoring, Dokumentation und Praxis}

In Modul 6 werden grundlegende Kenntnisse dazu vermittelt, wie unter Berücksichtigung der Therapieziele ein individualisiertes und multimodales Monitoring der MS nach festgelegten Standards durchgeführt werden sollte. Den Ausgangspunkt hierfür bildet ein detaillierter Blick auf die verschiedenen Ansätze des Monitorings bei MS sowie der Optimierung der Patient*innendokumentation, die in Form von Registern oder anderen Strukturen stattfinden sollte. Außerdem werden in diesem Modul auch die Anwendung und Interpretation von klassischen klinischen und bildgebenden Outcomes sowie „patient-reported outcomes" und weiteren speziellen Parametern aus dem eHealth-Bereich in Wissenschaft und Praxis thematisiert. Durch die intensive Auseinandersetzung mit verschiedenen Instrumenten zu MS-Dokumentation und -Management werden der Umgang mit und die Verwertung von den bei MS anfallenden großen Datenmengen (Real-World-Daten) geschult. Zudem werden gesundheitsökonomische Aspekte studiert, um Kostenarten unterscheiden zu können, Kostenanalysen durchzuführen und die Krankheitskosten bei MS aufzuschlüsseln. Im Weiteren beinhaltet Modul 6 die kritische Auseinandersetzung mit MS-spezifischen Netzwerken, Verbänden und Registern. Praxiserfahrungen in Monitoring, Patient*innenkommunikation und klinischer Dokumentation können in Fallkonferenzen gesammelt werden, die speziell das Monitoring und die Dokumentation thematisieren.

\section{Masterthesis bzw. wissenschaftliche Arbeit}

Unter individueller Betreuung der oder des Erstgutachtenden, der ein*e habilitierte*r Hochschullehrer*in aus dem Studiengang ist, wird eine Masterthesis bzw. ein wissenschaftliches Manuskript angefertigt, um den Transfer aus Fragestellungen der klinischen Praxis in die konkrete Patient*innenversorgung zu wissenschaftlichen Fragestellungen $\mathrm{zu}$ bewerkstelligen. Es kann zwischen der Prüfungsleistung Masterthesis oder wissenschaftliches Paper in einem dem Peerreview-Verfahren unterliegenden und in der Literaturdatenbank „PubMed“ gelisteten Journal gewählt werden. Ein Paper sollte als thematische Übersichtsarbeit, Metaanalyse oder als wissenschaftliche Originalarbeit gestaltet sein. Das Thema wird eingereicht und durch die wissenschaftliche Leitung des Studiengangs finalisiert.

\section{Lehrmethoden}

Die Lehrformen im Masterstudiengang „Multiple Sklerose Management" werden in definierten thematisch geprägten und strukturierten Modulen verwendet, womit einerseits die Abgeschlossenheit eines Themas sichergestellt, andererseits die Möglichkeit verbindender und übergreifender Themenbehandlung möglich ist. Die Lehrformen wurden sowohl als Präsenz- als auch Online-Veranstaltungen konzipiert. Vorlesungen, Tutorien, Fallkonferenzen und Journal Clubs sollen größtenteils online und teilweise in Präsenz stattfinden, wohingegen Hospitationen und Exkursionen sowie Kongressbesuche als Präsenzveranstaltungen geplant sind (Abb. 4).

Vorlesungen Die im Vordergrund stehende Lehrveranstaltungsform ist die diskursive Vorlesung, die der Wissensvermittlung dient sowie zur Mitarbeit anregen soll. Hier wird der Schwerpunkt auf die umfassende thematische Präsentation zum jeweiligen Thema

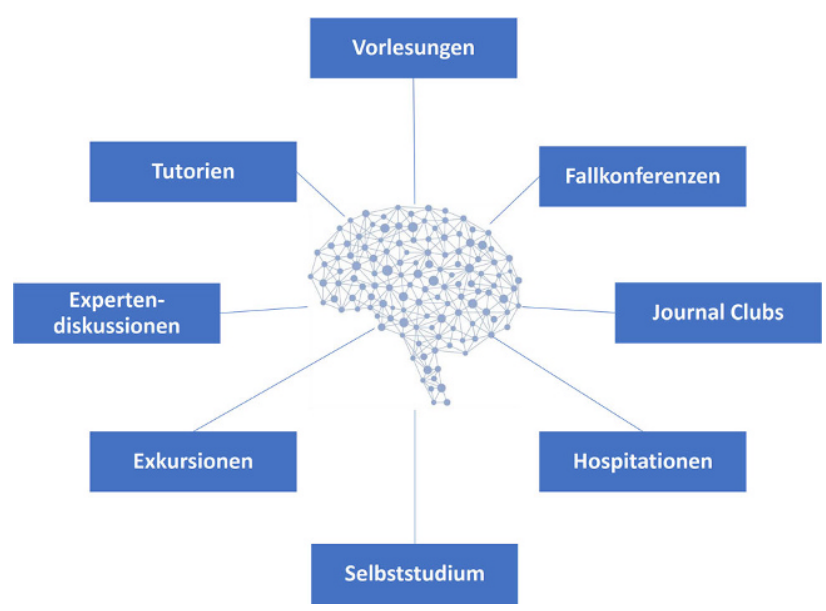

Abb. 4 Lehrmethoden im Studiengang „Multiple Sklerose Management" 
mit Bezug zur Praxis und Bedeutung für die zukünftige Tätigkeit als MS-Spezialist*in gesetzt. Auch auf die Aktualität der präsentierten Inhalte wird hingewiesen. Ein besonderes Anliegen stellt die Schaffung eines Wissens- und Erfahrungspools dar, der in die Lage versetzen soll, mit ärztlichen und nichtärztlichen Mitstreiter*innen in der jeweiligen Berufsgruppe auf „Augenhöhe“ zu debattieren, um dann gemeinsam beste Lösungsansätze zu finden. Dieser Anspruch setzt sich auch in weiteren Lehrmethoden durch und entwickelt sich bei näherer Betrachtung zu einer Art Alleinstellungsmerkmal der Studiengänge der DIU.

Tutorien Eine Besonderheit des Studiengangs sind die vor mündlichen Prüfungen stattfindenden Tutorien, in der der Unterrichtsstoff komprimiert aufbereitet wiederholt wird. Diese Veranstaltungen dienen nicht nur einer zweckmäßigen Prüfungsvorbereitung, sondern bieten zusätzlich eine sinnvolle, durch nochmals neue Aspekte geprägte Vertiefung der Lehrinhalte. Die erlangten Fachinhalte werden in eine bestehende medizinische, gesundheitspolitische und berufspolitische Wissenslandschaft eingeordnet.

Fallkonferenzen In Fallkonferenzen werden typische diagnostische und therapeutische Anwendungsprobleme anhand eines eigenen aufbereiteten Falls systematisch aufgearbeitet, diskutiert und präsentiert. Durch Einbindung von Expert*innen unterschiedlicher Spezialisierung z.B. im Bereich der klinischen Immunologie oder der Bildgebung kann das Wissen am konkreten Fall erweitert und direkt in der Betreuung der Patient*innen angewendet werden. Konkrete Patient*innenfälle werden mitsamt ihrer diagnostischen und differenzialdiagnostischen Komplexität sowie ihrem Krankheitsverlauf und ihren Therapieoptionen interpretiert. Dabei kommen in den Vorlesungen erlernte Inhalte zur Anwendung und können direkt unter Supervision angewendet werden. Die enorme Praxisnähe und praktische Relevanz macht diese Lehrform zu einer wichtigen Säule des Studiengangs.

Hospitationen Vor-Ort-Hospitationen in speziell ausgewählten Zentren mit großer Erfahrung in Diagnostik und Therapie erlauben eine direkte praktische Umsetzung der erlernten Inhalte vor Ort unter Supervision von erfahrenen Hochschullehrer*innen. Gerade die praktische Anwendung diagnostischer Verfahren bei individuellen Patient*innen sowie medikamentöser und nichtmedikamentöser Therapieverfahren kann somit direkt in der Praxis erlernt werden. Das Überprüfen der Zuverlässigkeit des Gelernten sowie die fachliche Auseinandersetzung mit „Praktiker*innen" verschafft nochmals Sicherheit bezüglich des Lernschatzes und eröffnet häufig neue fachliche und berufliche Perspektiven. Im verbalen Austausch mit kompetenten Expert*innen aus der Praxis kann das Erlernte nochmals reflektiert und vertieft und die eigene Form der Diskussion überprüft werden.

Expertendiskussionen und E-Journal-Clubs Wichtiges interaktives Element innerhalb des MS-Management-Studiums ist auch die hohe Aktualität der Lerninhalte und somit Umsetzung von ganz aktuellen wissenschaftlichen Publikationen in den klinischen Alltag. Daher werden im Rahmen des Studiengangs E-Journal-Clubs und Expert*innendiskussion durchgeführt. Dafür werden von erfahrenen, wissenschaftlich tätigen MS-spezialisierten Neurolog*innen rezente Publikationen vorgestellt, die in der Expert*innengruppe diskutiert werden, wobei deren Relevanz für den praktischen Alltag aufgezeigt werden soll. Die Teilnehmenden sind zur Diskussion aufgefordert. Damit ist es möglich, dass wissenschaftliche Innovationen nach kritischer Würdigung direkt in die klinische Praxis transferiert werden können.

Exkursionen und Kongressbesuche Zur Vermittlung von Erfahrungen im Bereich des MS-Managements werden Exkursionen und Expertengespräche mit beruflich Erfahrenen durchgeführt. Gerade auch die Übersetzung von wichtigen klinischen Fragestellungen in klinische Studien soll mithilfe der Kongressbesuche erlernt werden.

Selbststudium Ein effektives Selbststudium zur Wissensverbreiterung und -vertiefung wird durch eigenständiges Literaturstudium und zur Verfügung gestellte Lehr- und Lernunterlagen möglich. In jedem Modul werden umfangreiche Vorlesungsskripte, Buch- und Zeitschriftenbeiträge in Papierform oder elektronisch zur Verfügung gestellt. Diese Unterlagen ermöglichen es, den Lehrstoff individuell vorzubereiten, nachzuarbeiten und $\mathrm{zu}$ vertiefen sowie für die spätere eigene Tätigkeit aufzuarbeiten. Des Weiteren werden aktuelle Informationen (Prüfungsergebnisse, Präsenztermine etc.) im Download-Bereich des Campusmanagementsystems Campus Net zur Verfügung gestellt. Neben einem persönlichen Zugang zu Recherchediensten der Sächsischen Landesbibliothek, der Staats- und Universitätsbibliothek steht außerdem der Computerpool der DIU zur Verfügung.

Auf der Grundlage regelmäßiger studentischer Evaluationen der Lehreinheiten ist es jederzeit möglich, den Einsatz von Dozent*innen in bestimmten Modulen $\mathrm{zu}$ beurteilen, zu modifizieren oder zu korrigieren. Somit wird neben der fachlichen und beruflichen Qualifikation insbesondere auch den didaktischen Fähigkeiten der Dozent*innen eine besondere und entscheidende Bedeutung beigemessen.

\section{Bisherige Erfahrungen und Ausblick}

Die erste Matrikel mit 19 Studierenden durchläuft den Studiengang seit nunmehr 1 Jahr, welches bekanntermaßen ein sehr schwieriges Jahr war, denn 


\section{Infobox}

- Studiengang. Multiple Sklerose Management

- Studiendauer. 4 Semester

- Studienbeginn: jährlich im Frühjahr

- Studienvoraussetzungen: Hochschulabschluss, Abschluss in einem Gesundheitsfachberuf, mindestens einjährige einschlägige Berufserfahrung

- Studieninhalte: MS Grundlagen, MS Klinik und Diagnostik, MS Studien und Statistik, MS Therapie und Rehabilitation, MS Monitoring und Dokumentation

- Sprache: Deutsch, Englisch im Rahmen der Internationalisierung geplant

- Studiengebühren: 708€ monatlich

- Studienabschluss: Master of Science

- ECTS-Punkte: 60

- Schirmherrin: Deutsche Multiple Sklerose Gesellschaft (DMSG)

- Kooperationspartner: Deutsche Gesellschaft für Neurologie (DGN), Berufsverband Deutscher Nervenärzte (BVDN), Berufsverband Deutscher Neurologen (BDN), ParadigMS Foundation, European Multiple Sclerosis Platform (EMSP)

- Kontakt. DIU - Dresden International University, Freiberger Straße 37, 01067 Dresden, E-Mail: msm@di-uni.de

- Weitere Informationen: https://www.di-uni.de/ studium-weiterbildung/medizin/ms-manage ment

der Beginn des Masterstudiengangs fiel mit dem Ausbruch der COVID-19-Pandemie zusammen. So konnten die bestehenden Pläne nicht in vollem Umfang angewendet werden, und es mussten kurzfristig neue Konzepte im „Notfallmodus“ entworfen und umgesetzt werden. Ein Großteil der geplanten Veranstaltungen, bei denen dies möglich war, musste von Präsenzunterricht auf digitale Formate umgestellt werden, Vor-Ort-Veranstaltungen mussten verschoben werden. Eine erste Veranstaltung in Präsenz, ein Hospitationswochenende an der Universitätsklinik Göttingen, konnte mittlerweile im September 2021 stattfinden. Das Wochenende umfasste neben vielen Vorträgen und Besichtigungen einen MikroskopiePraxisworkshop Pathogenese und Pathologie am Neuropathologischen Institut unter Leitung von Prof. Dr. Christine Stadelmann-Nessler sowie einen weiteren immunologischen Workshop zum Thema „Ak-Diagnostik per FACS“ unter Leitung von Dr. Stefan Nessler. Am letzten Tag erhielten die Studierenden konkrete Organisationseinblicke in die MS Spezialambulanz in Göttingen. Das nächste Hospitationswochenende ist im März 2022 im Neurologischen Rehabilitationszentrum Quellenhof unter fachlicher Leitung von Prof. Dr. Peter Flachenecker geplant. An drei Tagen werden Kleingruppenhospitationen und ein Workshop zu pal- liativmedizinischen Aspekten mit Prof. Dr. Raymond Voltz stattfinden. Neben zwei weiteren Klinikbesuchen in Würzburg unter Leitung von Prof. Dr. Mathias Mäurer und in Düsseldorf unter Leitung von Prof. Dr. Sven G. Meuth ist ein weiteres Hospitationswochenende am Universitätsklinikum Dresden geplant. An diesem Wochenende werden die Studierenden das Dresdner MS-Zentrum kennenlernen und in einer Mischung aus theoretischen Wissens- und praktischen Hands-on-Einheiten die Themen Ganganalyse, Neuroophthalmologie, optische Kohärenztomographie (OCT), Infusionsmanagement, Magnetresonanztomographie (MRT) und neuropsychologische Testungen vertiefen. Alle anderen Veranstaltungen finden nach wie vor online statt. Hier gelang es der DIU in kurzer Zeit, Microsoft Teams als cloudbasierten digitalen Hub und technische Basis sowie als zentrale Lehr-, Lern-, Kommunikations- und Kooperationsplattform $\mathrm{zu}$ etablieren, was sich als sehr effektiv erwies. So standen Online-Klassenzimmer in einer Fernstudienumgebung für synchrones und selbstgesteuertes asynchrones Lernen zur Verfügung. Die Einrichtung eines Lernvideoportals und spezieller Lernbereiche für Peer-to-Peer-Lernen ermöglicht einen flexiblen Wissenstransfer. Insgesamt kann festgehalten werden, dass der Masterstudiengang von der Digitalisierung profitierte - nicht nur beim Lernen und Lehren, sondern auch durch die Möglichkeiten der engen Abstimmung zwischen den Dozent*innen und der Studiengangsleitung mit der Hochschulleitung und den Modulkoordinator*innen sowie den Studierenden untereinander.

Teil der Qualitätssicherung des Studiengangs ist die Evaluation der Veranstaltungen durch die Studierenden. Die Ergebnisse der Evaluation der ersten beiden Module des Programms liegen bereits vor, die anderen Module werden ebenfalls bewertet. Die Studierenden gaben den bisher bewerteten Einzelkursen in den Modulen und den Modulen insgesamt gute bis sehr gute Noten. Sie waren sehr zufrieden mit den Inhalten der Lehrveranstaltungen, mit der Wissensvermittlung durch die Dozent*innen und mit der Interaktion untereinander sowie mit den Dozent*innen. Einige der Studierenden wünschten sich mehr Zeit für bestimmte Themen, mehr Interaktion mit den Dozent*innen und hätten gerne einige spezielle Themen wie die Magnetresonanztomographie (MRT) vor Ort in einer Präsenzveranstaltung behandelt. Solche im Studiengang fest verankerten Präsenzveranstaltungen werden hoffentlich in naher Zukunft häufiger wie geplant stattfinden können [16].

Aufgrund dieser äußerst positiven Erfahrungen ist eine Internationalisierung des Programms in Kooperation mit der European Charcot Foundation geplant, um Neurolog*innen und Interessierten aus anderen Ländern den Zugang zu diesem hochwertigen Masterstudiengang zu ermöglichen. Der Studiengang wird dann in englischer Sprache angeboten werden. 
Interessenkonflikt C. Stadelmann erhielt Honorare für Vorträge und Beratungstätigkeiten von Bayer, Roche, Novartis und Merck. S.G. Meuth erhielt Vortragshonorare und Reisekosten für die Teilnahme an Konferenzen von Almirall, Amicus Therapeutics Germany, Bayer Health Care, Biogen, Celgene, Diamed, Genzyme, MedDay Pharmaceuticals, Merck Serono, Novartis, Novo Nordisk, ONO Pharma, Roche, SanofiAventis, Chugai Pharma, QuintilesIMS und Teva. Er erhielt Forschungszuschüsse von: Bundesministerium für Bildung und Forschung (BMBF), Deutsche Forschungsgesellschaft (DFG), Else-Kröner-Fresenius-Stiftung, Deutscher Akademischer Austauschdienst, Hertie Stiftung, Interdisziplinäres Zentrum für Klinische Forschung (IZKF) Münster, Deutsche Stiftung Neurologie und Almirall, Amicus Therapeutics Germany, Biogen, Diamed, Fresenius Medical Care, Genzyme, Merck Serono, Novartis, ONO Pharma, Roche und Teva. P. Flachenecker erhielt Honorare für Vorträge und Beratungstätigkeiten von den Firmen Almirall, Bayer, Biogen Idec, BMSCelgene, Coloplast, Genzyme, Hexal, Janssen-Cilag, Novartis, Merck Serono, Roche, Stadapharm und Teva. M. Mäurer erhielt Honorare für Vorträge und Beratungstätigkeiten von den Firmen Almirall, Alexion-AstraZeneca, Bayer, Biogen Idec, BMS-Celgene, Genzyme, GlaxoSmithKline, JanssenCilag, Novartis, Merck Serono, Roche, und Teva. R.H.W. Funk, F. Ramisch und J. Niemeier sind Mitglieder der DIU, die das Programm veranstaltet. T. Ziemssen erhielt eine persönliche Vergütung von Biogen, Bayer, Celgene, Novartis, Roche, Sanofi und Teva für Beratungsdienste und zusätzliche finanzielle Unterstützung für die Forschungsaktivitäten von Bayer, BAT, Biogen, Novartis, Teva und Sanofi. I. Voigt und B. Schipp geben an, dass kein Interessenkonflikt besteht.

\section{Literatur}

1. Compston A, Coles A. Multiple sclerosis. Lancet. 2008; 372(9648):1502-17.

2. Ziemssen T. Symptom management in patients with multiple sclerosis. J Neurol Sci. 2011;311(Suppl 1):S48-S52.

3. Giovannoni G. Disease-modifying treatments for early and advanced multiple sclerosis: a new treatment paradigm. Curr Opin Neurol. 2018;31(3):233-43.

4. ThompsonAJ, Banwell BL, BarkhofF, CarrollWM, Coetzee T, Comi G, et al. Diagnosis of multiple sclerosis: 2017 revisions of the McDonald criteria. LancetNeurol.2018;17(2):162-73.

5. The University of Sheffield. Postgraduate study: neuroscience and neurodegeneration. 2021. https:/ / www.sheffield. ac.uk/postgraduate/taught/courses/2021/neuroscienceand-neurodegeneration-msc?utm_source=findamasters\& utm_campaign=courseid, Sheffield. Zugegriffen: 23. Juli 2021.
6. Multiple Sclerosis Center of Catalonia/Vall Hebron Campus. Master in neuroimmunology. 2021. https://www. cem-cat.org/en/corse/master-neuroimmunology, Barcelona. Zugegriffen: 23. Juli 2021.

7. Universität Basel. MAS Neurophysiotherapie - Fachexperte/Fachexpertin in Multiple Sklerose, Morbus Parkinson und Stroke. 2021. https://advancedstudies.unibas.ch/ studienangebot/kurs/mas-neurophysiotherapie--fachex pertefachexpertin-in-multiple-sklerose-morbus-parkinson-und-stroke-206474, Basel.Zugegriffen:23. Juli 2021.

8. Deutsche Multiple Sklerose Gesellschaft Bundesverband. MS-Schwester/MS-Therapiemanagement: DMSG Fachfortbildung für „MS-Schwestern“. 2021. https://www. dmsg.de/service/fachfortbildungen/ms-schwester/, Hannover.Zugegriffen: 23. Juli 2021.

9. The British MS Healthcare Professionals Society Sheffield. Multiple sclerosis academy. 2021. https://www.sheffield. ac.uk/postgraduate/taught/courses/2022/neuroscienceand-neurodegeneration-msc?utm_source=findamasters \& utm_campaign=courseid, Sheffield. Zugegriffen: 23 . Juli 2021 .

10. Dresden International University. Multiple Sklerose Management (M.Sc.). 2021. https://www.di-uni.de/studiumweiterbildung/medizin/ms-management, Dresden.Zugegriffen:23. Juli 2021.

11. Medizinische Fakultät, TU Dresden. Neuer DIU-Studiengang: Multiple Sklerose Management. 2019. https:// tu-dresden.de/med/mf/die-fakultaet/newsuebersicht/ neuer-diu-studiengang-multiple-sklerose-management. Zugegriffen: 1.Sept.2019.

12. Ziemssen T. Master im Multiple-Sklerose-Management. DNP. 2019;20(4):15.

13. Eichstädt S. MS-Management: Neuer Studiengang in Dresden.Ärztezeitung, Bd. 81-137D. 2019.

14. Voigt I, Ziemssen T. New master's program: multiple sclerosis management. Multiple Scler J. 2019;25(2_suppl):131-356.

15. DMSG. Berufsbegleitender Masterstudiengang MS Management.2020.https://www.dmsg-berlin.de/de/aktuelles/ detailansicht/berufsbegleitender-masterstudiengangms-management/kt/1/.Zugegriffen:23. Juli2021.

16. Voigt I, Stadelmann C, Meuth SG, Funk RHW, Ramisch F, Niemeier J, et al. Innovation in digital education: lessons learned from the multiple sclerosis management master's program. Brain Sci. 2021;11(8):1110.

Hinweis des Verlags Der Verlag bleibt in Hinblick auf geografische Zuordnungen und Gebietsbezeichnungen in veröffentlichten Karten und Institutsadressen neutral. 